\title{
Análise da utilização das tecnologias digitais por diretores escolares e professores
}

\author{
João Piedade \\ Neuza Pedro \\ Instituto de Educação \\ Universidade de Lisboa (Portugal)
}

\section{Resumo}

presente artigo apresenta os resultados de um estudo desenvolvido em torno da utilização das tecnologias digitais em contexto escolar, em particular, por parte de diretores escolares e professores do ensino básico e secundário Português. Procurou-se, deste modo, analisar os índices de utilização das tecnologias digitais e os níveis de proficiência na utilização das mesmas apresentados pelos diretores e pelos professores nas suas práticas. Organizado com base numa abordagem metodológica de cariz quantitativo, e assumindo uma natureza descritiva e exploratória, o estudo envolveu a recolha de dados junto de 133 diretores escolares e 1908 professores através da aplicação de três escalas self-report. Os resultados reportaram que os diretores escolares apresentam índices médios favoráveis de autoeficácia e de utilização das tecnologias nas suas práticas profissionais. Evidenciaram, igualmente, níveis elevados de utilização das tecnologias por parte dos professores. A análise, cruzando os resultados dos diretores com os dos professores, permitiu agrupar as escolas com base em diferentes patamares considerando o nível de proficiência evidenciada pelos seus diretores e detetar que, no seio destas, a utilização das tecnologias evidenciada pelos seus professores se revela semelhante ao nível de proficiência revelado pelo respetivo diretor.

Diretores escolares. Professores. Utilização educativa das tecnologias.

\section{Analysis of digital technologies use by school principals and teachers}

\section{Abstract}

This paper presents the results of a study developed in the scope of the use of digital technologies in school context by school principals and teachers in primary and secondary Portuguese education. The aim was to analyze the utilization levels of digital technologies and the levels of proficiency in their use presented by the school principals and the teachers. Organized on a descriptive and exploratory quantitative methodological approach, the study involved the data collection from 133 school principals and 1908 teachers through the application of three self-report scales. The results reported that school principals present favorable average scores of proficiency and use of the technologies in their professional practices. They also showed high levels of use of the technologies by teachers. The analysis, crossing the results of the principals with the teachers, allowed to distinguish the schools based on different levels of proficiency evidenced by their principals and to detect that within the latter, the use of the technologies evidenced by their teachers is similar to level of proficiency revealed by the respective principals.

Keywords: School principals. Teachers. Technology uses in education. 


\section{Análisis de la utilización de las tecnologías digitales por directores escolares y profesores}

\section{Resumen}

Este artículo presenta los resultados de una investigación diseñada en torno al uso de las tecnologías digitales en las escuelas, en particular, por los directores y profesores de las escuelas de Portugal. Así se buscó analizar los índices de utilización de las tecnologías digitales y los niveles de competencia en su utilización presentados por los directores y los profesores en sus prácticas de trabajo. Organizado sobre un enfoque metodológico cuantitativo, y asumiendo una naturaleza descriptiva y exploratoria, el estudio involucró la recogida de datos en 133 directores escolares y 1908 profesores a través de la aplicación de tres escalas self-report. Los resultados indicaron que los directores escolares presentan niveles medios favorables de competencia y de utilización de las tecnologías en sus prácticas de trabajo. También evidenciaron niveles elevados de utilización de las tecnologías por parte de los profesores. El análisis, cruzando los resultados de los directores con los resultados de los profesores, permitió organizar las escuelas con base en diferentes niveles de competencia evidenciada por sus directores y detectar que, en estas escuelas, los niveles de utilización de las tecnologías evidenciados por sus profesores se revelan semejante al nivel de competencia revelado por el director. Palabras claves: Directores escolares. Profesores. Utilización educativa de las tecnologías.

\section{Introdução}

O presente estudo, realizado no âmbito do programa doutoral em educação do Instituło de Educação da Universidade de Lisboa, enquadra-se no domínio de especialidade das tecnologias da informação e comunicação, assume como foco a utilização das tecnologias digitais (TD) nas práticas profissionais dos diretores escolares e dos professores do ensino básico e secundário Português.

Atendendo às premissas anteriores, esta investigação empírica, de natureza quantitativa, procura contribuir para a análise dos i) níveis de proficiência e ii) níveis de utilização das tecnologias nas práticas profissionais dos diretores de escolas ou agrupamento de escolas do ensino público português. Procurou-se ainda, analisar os níveis de utilização das TD apresentados pelos professores nas várias dimensões da sua prática profissional, procurando, adicionalmente explorar a existência de algum tipo de relação entre o nível autoeficácia e de utilização das tecnologias pelos diretores escolares e o nível de utilização das mesmas pelos professores. Deste modo, os participantes 
Análise da utilização das tecnologias digitais por diretores escolares e professores

desta pesquisa são os diretores escolares e professores do ensino básico e secundário de escolas públicas de Portugal continental.

Considerou-se pertinente analisar o sentido de proficiência e as práticas dos diretores escolares na utilização das tecnologias, pelo papel que aos mesmos se atribui na literatura enquanto promotores de processos de integração das tecnologias nos seus contextos escolares e, em particular, nas práticas profissionais dos seus professores.

A recolha de dados foi organizada com base na aplicação de três escalas self-report organizadas num único instrumento disponibilizado online, i) a escala de proficiência na utilização da TD, e ii) a escala de utilização das tecnologias digitais nas práticas de gestão escolar aplicadas aos diretores escolares, e iii) a escala de utilização das tecnologias digitais nas práticas docentes aplicada aos professores.

Considerando que a investigação em contexto português tem assumido como público prioritário os professores, este estudo ambicionou contribuir para a produção de conhecimento científico sobre a proficiência e a utilização das tecnologias digitais nas práticas profissionais dos diretores escolares, que têm vindo a ser negligenciados em várias das iniciativas de promoção do uso das tecnologias no contexto escolar.

\section{Tecnologias digitais na escola: papéis, proficiência e atitudes dos diretores escolares relativamente à utilização das tecnologias digitais}

Os diretores escolares, enquanto entidades máximas das escolas, com responsabilidades ao nível da gestão e administração, mas também na definição de planos e estratégias de intervenção a nível pedagógico, têm um papel preponderante nos processos de integração das tecnologias digitais nos seus contextos escolares. Neste enquadramento teórico, num primeiro momento começa-se por analisar a importância e o papel dos diretores na promoção da utilização pedagógica das tecnologias nas práticas escolares. Num segundo foco de análise discute-se a utilização das tecnologias digitais por parte dos diretores escolares. 
Os estudos realizados, em contexto português, sobre o papel dos diretores escolares nos processos de utilização das tecnologias em contexto educativo são escassos. Esta ausência de estudos em contexto nacional poderá ser justificada pela escassez de iniciativas de formação na área das tecnologias digitais tendo como público-alvo os diretores escolares. As iniciativas e programas neste domínio têm-se destinado, nos últimos anos, maioritariamente aos professores do ensino básico e secundário.

Em sentido oposto, em contexto internacional vários têm sido os estudos que se têm dedicado a analisar e produzir evidência sobre o importante papel dos diretores escolares na promoção da integração educativa das tecnologias e da modernização das práticas escolares (ABDULLAH, DEWITT, ALIAS, 2013 ; AFSHARI, BAKAR, LUAN, AFSHARI, SAY, FOOI, 2010; CAKIR, 2012; CAKIR, 2014 ; DRENT, MEELISSEN, 2008; KHALID, BUUS, 2014; MAKEWA, MEREMO, ROLE, ROLE, 2013; MOOLENAAR, SLEEGERS, 2015; SEYAL, 2015; STUART, MILLS, REMUS, 2009; TONDEUR, KEER, BRAAK, VALCKE, 2008; WONG, KHADIJAH, 20171

Autores como Jacob e Hunter (2004) e Stuart, Mills e Remus (2009) referem que os diretores escolares se encontram sobre uma crescente pressão para a integração das tecnologias nas suas escolas, e que os mesmos devem ser os responsáveis por definir as medidas necessárias para que a modernização das práticas educativas se efetive nos seus contextos escolares. A liderança escolar exercida pelos diretores tem vindo a ser recorrentemente referida em várias investigações como um dos fatores potenciadores da utilização das tecnologias em contexto educativo. Alguns autores têm apontado que, mais importante que a infraestrutura tecnológica parece ser a liderança e o suporte que esta pode providenciar o fator que apresenta maior preponderância na efetiva modernização das práticas escolares suportada por tecnologias digitais (ANDERSON, DEXTER, 2005; CALABRESE, 2002; SCHILLER, 2003). Na mesma linha de pensamento, Asthari, Bakar, Luan, Asthari, Say e Foy (2010) apontam a figura do diretor escolar como fator crítico para a transformação tecnológica do currículo escolar, sugerindo a necessidade de ver desenvolvidos programas de formação em liderança transformacional, gestão e tecnologia dirigidos aos diretores escolares. Vários outros autores referem, igualmente, que o diretor escolar deve assumir uma liderança transformacional capaz de promover o envolvimento e compromisso por parte da sua comunidade escolar na direção a uma efetiva adoção e utilização das tecnologias digitais (KHALID, 
Análise da utilização das tecnologias digitais por diretores escolares e professores

BUUS, 2014; SEYAL, 2015; WONG, LI, 2008). Cakir (2012, p. 279) enuncia que, no âmbito das várias responsabilidades que os diretores escolares assumem, a 'liderança tecnológica' (technology leadership) deve ser, atualmente, uma prioridade. Refere o autor, "[...] being a technology leader should entail responsibilities such as acting as a role model, providing encouragement and direction, sharing knowledge and information and [...] they are also expected to be highly proficient in using technology".

Abdullah, DeWitt e Alias (2013) salientam em especial a importância das crenças pessoais dos diretores escolares enquanto fatores de influência na adoção e implementação das tecnologias digitais nas suas escolas. Deste modo, apontam os mesmos autores que, os diretores escolares com uma forte visão estratégica sobre a importância das tecnologias digitais na promoção de atividades pedagógicas inovadoras, assumem um papel ativo e preponderante na criação de uma cultura de utilização das tecnologias nos seus contextos escolares. A importância das crenças e atitudes pessoais é, igualmente, referida por Cakir (2014, p. 736) ao indicar que "[...] the success of any attempt to implement technology in educational programs depends strongly upon the support and attitudes of teachers and principals". Conclui o mesmo autor, no seu 5 estudo com 460 professores e 308 diretores de escolas turcas, que as crenças e atitudes dos professores e dos diretores escolares têm grande influência sobre a utilização das tecnologias digitais por parte destes agentes educativos.

Constata-se assim que, o papel dos diretores escolares no processo de integração das tecnologias em contexto educativo tem vindo a ser referido, por vários autores, como um fator importante a considerar. Jacobson e Hunter (2004) referiam que apesar da inovação educacional implicar um esforço conjunto de toda a comunidade escolar, o papel dos diretores é imprescindível. Esta mesma importância é reconhecida por Stuart, Mills e Remus (2009) quando referem que compete aos líderes escolares a definição das estratégias de mudança que se revelam necessárias nos seus contextos educativos, e que para que essa mudança se instale o seu papel é preponderante. Na mesma linha de argumentação surgem outros estudos desenvolvidos recentemente (CAKIR, 2012; KHALID, BUUS, 2014 ; PELED, KALID, DORI, 2011 ; SEYAL, 2015 ).

Considerando a importância do papel dos diretores escolares, na efetiva utilização das tecnologias nos seus contextos escolares a investigação refere a necessidade de os diretores serem competentes e proficientes na utilização das tecnologias, acredita-se que só deste modo estes podem assumir uma 
verdadeira liderança tecnológica. A crença que os diretores possuem sobre a sua proficiência ou competência na atuação com as tecnologias digitais é assim fundamental para o suporte e o incentivo que podem proporcionar aos seus professores na modernização das práticas escolares. Considera-se assim que, os diretores devem procurar envolver-se em programas de desenvolvimento profissional que lhes permitam desenvolver as competências necessárias para impulsionarem estes processos de inovação nos seus contextos escolares (AFSHARI; GHAVIFEKR; SIRAJ; SAMAD, 201 2), nomeadamente os tecnológicos.

Os diretores escolares do séc. XXI estão perante enormes desafios proporcionados pelas tecnologias digitais (GRAY; SMITH, 2007) e, deste modo, precisam de rever as suas atitudes, proficiência, perceções e níveis de utilização das tecnologias nas suas práticas profissionais (DAY; LEITHWOOD, 2007). As tecnologias podem proporcionar um conjunto de potencialidades que permitem incrementar a performance dos diretores (MAKI, 2008; ZAINALLY, 2008). Trazem novos benefícios para todo o contexto escolar e, em particular, para as atividades desenvolvidas pelos diretores escolares, em áreas como a comunicação, a gestão de processos de alunos e professores, a avaliação, a planificação, a gestão administrativa e financeira, entre outras.

Saiti e Prokopiadou (2009), num estudo realizado em escolas gregas sobre o impacto das tecnologias digitais na administração escolar, referem que a utilização das tecnologias no contexto escolar pode ser sinónimo de modernização e inovação nessas mesmas práticas. Estes autores mencionam que as tecnologias digitais proporcionam um conjunto de ferramentas online que podem facilitar as transações administrativas e tornam mais eficientes os processos de partilha de informação entre a comunidade educativa.

Procurando analisar as competências e conhecimentos que os diretores escolares consideram possuir na utilização das tecnologias, Asthari, Bakar, Luan, Asthari, Say e Foy (2010) levaram a cabo uma investigação com 320 diretores de escolas iranianas. Os resultados evidenciaram níveis de competência positivos ( $M=2.99$ ) no score global da escala utilizada, sendo que os scores mais elevados foram verificados nas operações básicas usando o computador $(M=3.60)$, utilização do processador de texto $(M=3.51)$ e telecomunicações $(M=3.19)$. Os scores mais baixos foram verificados na utilização de folhas de cálculo $(M=2.44)$ e bases de dados $(M=2.45)$, sendo ainda assim favoráveis, considerando a escala entre 1 a 5 pontos. Complementarmente, Cakir (2012) verificou num estudo desenvolvido com 21 diretores e 17 assistentes de diretor, 
Análise da utilização das tecnologias digitais por diretores escolares e professores

que os diretores apresentam uma forte utilização de aplicações de processamento de texto e folha de cálculo, e em sentido oposto, aponta que nenhum dos diretores inquiridos referiu usar ou estar familiarizado com tecnologias da web 2.0 (plataformas, blogs, wikis, RSS, etc.).

Na mesma linha de investigação Hoque, Razak e Zohora (2012) procuraram conhecer as práticas de utilização das tecnologias da informação e comunicação por professores $(n=215)$ e diretores $(n=45)$ de escolas da Malásia. $\bigcirc$ estudo procurou obter dados sobre três aspetos: al políticas de incentivo à implementação das tecnologias; b) disponibilidade e acessibilidade a recursos TIC; e c) eficiência e capacidade de utilização das tecnologias. Focando o último ponto, os dados revelaram que a utilização das tecnologias na gestão escolar, nas escolas analisadas, é ainda muito limitada e que, de um modo geral, as tecnologias não são usadas de forma efetiva e eficiente. Os autores salientam ainda a inexistência de políticas de incentivo, desenvolvidas por parte do Ministério da Educação da Malásia, que possam suportar e tornar efetiva a utilização das tecnologias nos contextos escolares.

Cakir (2014), num estudo desenvolvido junto de 460 professores e 308 diretores do ensino pré-escolar da Turquia, procurou avaliar o interesse, a ansiedade e a utilização das tecnologias nas práticas profissionais de diretores e professores. $\bigcirc$ autor concluiu que professores e diretores apresentam valores elevados na variável utilização das tecnologias e níveis mais baixos na variável ansiedade. Refere ainda que estes resultados se justificam pelo facto de que quanto mais elevados forem os índices de utilização das tecnologias, menor será o índice de ansiedade perante as mesmas.

Em 2015, Gastelú, Kiss e Dominguez desenvolveram um estudo, em que participaram cerca de $20 \%$ dos diretores escolares de escolas básicas de Veracruz no México, em que pretenderam analisar as atitudes dos diretores perante os computadores e o seu nível de atuação com os mesmos. Relativamente à utilização dos computadores pela comunidade escolar, 74\% dos participantes referiu que estes equipamentos são usados pelos diretores escolares, 60\% referiu que são usados também pelos professores, 37\% referiu que são usados pelos estudantes e $9 \%$ que são usados pelo restante staff da escola. Os autores referem, igualmente, que os diretores escolares inquiridos apresentam uma atitude positiva relativamente às tecnologias e, que as conseguem utilizar com regularidade na gestão de tarefas diárias. No entanto, concluem que, apesar dos resultados, "[...] integration process of ICT in public 
primary schools in Veracruz city is a pending task to be resolved" (GASTELÚ; KISS; DOMINGUEZ, 2015, p. 822), sinalizando que se revela necessário providenciar infraestruturas tecnológicas, desenvolver planos de formação para diretores e professores e desenhar políticas educativas nacionais que promovam a presença das tecnologias nas atividades das escolas.

Concluindo, fica claro que os diretores escolares têm um papel preponderante no processo de modernização de práticas nos seus contextos escolares, em particular na efetiva integração das tecnologias digitais. As suas atitudes, proficiência na utilização, visão estratégica, bem como o papel de liderança tecnológica exercido, são apresentados na literatura como fatores relevantes e potenciadores da utilização das tecnologias nas escolas. Pelo exposto na literatura considerou-se pertinentes analisar, no contexto português, a proficiência e a utilização das tecnologias digitais pelos diretores escolares, procurando igualmente analisar os índices de utilização pelos professores nas suas práticas.

\section{Design metodológico}

Considerando os objetivos assumidos desenvolveu-se uma investigação empírica de natureza quantitativa, que teve como propósito compreender e explicar a relação entre variáveis e contribuir para encontrar regularidades e orientações explicativas da forma de comportamento dos construtos em análise (ALMEIDA; FREIRE, 2008). O estudo em causa, enquadrou-se assim, num paradigma de investigação pós-positivista (CRESWELL, 2010), garantindo-se por parte do investigador uma postura objetiva e não intervencionista, orientando a ação para a procura e garantia de padrões adequados de validade, fidedignidade e fiabilidade para com os dados obtidos e as conclusões apresentadas (CRESWELL, 2010). De acordo com Maroco (201 1), o estudo pode ser, igualmente, classificado como uma investigação correlacional com finalidade prospetiva, em que a informação sobre uma dada população é inferida através de uma amostra representativa dessa mesma população e onde com esta se pretendem criar modelos de cabal poder explicativo no fenómeno em análise.

A nossa investigação baseou-se na obtenção de indicadores numéricos quantificáveis representativos das variáveis em análise, a saber:

- $\quad$ sentido autoeficácia na utilização das tecnologias digitais operacionalizado através do score total médio obtido na escala psicométrica selecionada 
para o efeito, a Computer Self-efficacy Scale desenvolvida por Cassidy e Eachus (2002).

- $\quad$ utilização das tecnologias digitais nas práticas de gestão escolar operacionalizada através do score total médio obtido na escala de utilização das TD nas práticas de gestão escolar desenvolvida pelos investigadores no âmbito deste estudo.

- $\quad$ utilização das tecnologias digitas nas práticas docentes operacionalizada através do score total médio obtido na escala de utilização das tecnologias nas práticas profissionais dos professores, a Teacher's Technology Use Scale desenvolvida por Bebell, Russell e O’Dwyer (2004).

\section{Instrumentos de recolha de dados}

A Computer Self-efficay Scale foi desenvolvida por Cassidy e Eachus (2002) considerando os princípios da teoria social e cognitiva de Bandura. A escala originalmente é composta por 30 itens, com 5 opções de resposta de formato tipo-likert, variando entre "Discordo Totalmente" e "Concordo Totalmente", sendo que 15 itens são formulados de forma positiva e 15 itens de forma negativa. Nos itens formulados de forma negativa, a cotação das respostas foi feita de forma inversa. Neste estudo, utilizou-se uma versão reduzida da escala, composta por 27 itens, resultante do seu processo de validação numa investigação realizada em contexto português (PEDRO, 2011 1).

A escala de utilização das tecnologias digitais nas práticas de gestão escolar, desenvolvida no âmbito do presente estudo, apresenta-se como um instrumento multidimensional, com o qual se pretendeu analisar a utilização das tecnologias pelos diretores em várias dimensões da sua atividade profissional. $\bigcirc$ instrumento desenvolvido é constituído por 22 itens com 5 opções de resposta, variando entre "Muito Raramente" e "Muito Frequentemente". Os 22 itens foram organizados em 5 dimensões, a saber:

1) Comunicação: frequência de utilização das tecnologias pelos diretores para comunicação com os vários elementos da sua comunidade educativa, constituída pelos itens P1 , P2, P3, P4, P5 e P10;

2) Planificação: frequência de utilização das tecnologias para planificação e preparação da atividade profissional, constituídas pelos itens P6, P7, P8 e P1 3; 
3) Dinamização de Reuniões: frequência de utilização das tecnologias para realização e dinamização de reuniões, constituída pelos itens P9, P1 1, Pl 4 e P22;

4) Avaliação: frequência de utilização das tecnologias nos processos e avaliação de pessoal docente, não docente e autoavaliação de escola, constituída pelos itens P12, P15, P16 e P18;

5) Gestão: frequência de utilização das tecnologias para realização de atividades de gestão e administração, constituída pelos itens P17, P19, P20 e P21;

Por último, a Measure Teacher's Technology Use Scale desenvolvida por Bebell, Russel e O'Dwyer (2004) é apresentada pelos autores como um instrumento de estrutura multidimensional, que pretende medir a utilização das tecnologias digitais em diferentes dimensões das práticas profissionais dos professores. Os autores consideram que a utilização das tecnologias ocorre com diferentes intensidades em diferentes atividades da profissão docente, pelo que organizaram o instrumento em sete dimensões, integrando no total 24 itens, a saber:

1) Preparação (Preparation): utilização das tecnologias pelos professores na organização e preparação das atividades de ensino-aprendizagem, constituída pelos itens Q1, Q2 e Q6;

2) E-mail profissional (Professional E-mail): utilização profissional do e-mail pelos professores para contacto com alunos, outros professores, órgãos de gestão e encarregados de educação, constituída pelos itens Q3, Q4, Q8 e Q24;

3) Instrução (Delivering Instruction): utilização das tecnologias pelos professores para o desenvolvimento de atividades instrucionais em sala de aula, constituída pelos itens Q7, Q9 e Q14;

4) Adaptação (Accommodation): utilização das tecnologias pelos professores para adaptação de recursos e atividades às necessidades educativas dos alunos, constituída pelos itens Q5, Q10 e Q13;

5) Uso pelos alunos (Student Use): integração das tecnologias em sala de aula para utilização pelos alunos no desenvolvimento e realização das atividades propostas pelo professor, constituída pelos itens Q12, Q15, Q16 e Q20;

6) Suporte à produção dos alunos (Student Products): utilização das tecnologias pelos alunos na produção de recursos requeridos pelo professor, constituída pelos itens Q18, Q19, Q21 e Q22; 
Análise da utilização das tecnologias digitais por diretores escolares e professores

7) Avaliação (Grading): utilização das tecnologias pelos professores no processo de cálculo, registo e atribuição de classificações, constituída pelos itens Q11, Q17 e Q23;

Os 24 itens apresentam-se em formato de escala, constituídos por perguntas de avaliação ou estimação, que solicitam aos professores que indiquem a resposta a cada item, tendo por base a escala apresentada, de "Muito Raramente" a "Muito Frequentemente", sendo cotada com valores entre 1 e 5 respetivamente.

Após o processo de construção e/ou adaptação dos instrumentos de recolha de dados revelou-se necessário avaliar a sua qualidade métrica através da análise dos indicadores de sensibilidade, validade e fiabilidade IMAROCO, 201 1), procurando, desta forma, eliminar quaisquer itens dos instrumentos que se relassem menos discriminativos.

A análise da consistência interna da escala Computer Self-efficacy Scale foi previamente analisada em dois outros estudos nacionais, tendo a mesma apresentado um elevado índice de consistência interna em ambos com alpha de cronbach de .94 e .93 respetivamente (PEDRO, 2011 ; PIEDADE, $112010)$. Com o objetivo de confirmar os resultados encontrados nos dois estudos prévios, procedeu-se à análise da consistência interna da escola considerando os 133 diretores. Os dados revelaram, igualmente, um elevado índice de consistência interna $(\alpha=.90)$.

Após o processo de desenvolvimento da escala de utilização das tecnologias na gestão escolar procedeu-se à sua validação por especialistas e à sua pré-testagem junto de um grupo de diretores escolares. Através dos contributos de três académicos especialistas na área da gestão e administração escolar procedeu-se à reformulação de 2 itens e à eliminação de um outro, originado assim a segunda versão da escala. Num segundo momento, procedeu-se á validação da segunda versão junto de diretores escolares experientes no desempenho do cargo. A testagem junto deste grupo baseou-se na análise do critério de fidelidade. A análise das respostas dos 10 diretores revelaram um elevado índice de consistência interna ( $\alpha=$.97). A análise da qualidade métrica da escala e os comentários e sugestões dos diretores levaram à reformulação dos itens 1, 13, 17 e 21, originando desta forma a versão final do instrumento. Com o objetivo de comprovar os resultados encontrados na pré-testagem procurou-se testar novamente a qualidade métrica com base nas respostas dos 
133 diretores participantes. Através da análise fatorial com recurso ao método de componentes principais, rotação Varimax, definindo à partida 5 fatores fixos que corresponde às 5 dimensões do instrumento, foi possível verificar que os 5 fatores explicavam cerca de $73 \%$ da variabilidade total da escala. A análise da fiabilidade da escala permitiv encontrar valores indicativos de uma elevada consistência interna para a mesma $(\alpha=$.94).

Por último procedeu-se a análise da qualidade métrica da Measure Teacher's Technology Use Scale composta por 24 itens organizados em cinco dimensões. Num primeiro foco de análise começou-se pela aplicação do modelo de análise fatorial confirmatória com o objetivo de perceber se o modelo definido pelos autores (BEBELL; RUSSEL; O'DWYER, 2004) se confirmava na amostra constituída pelos 1908 professores do ensino básico e secundário. A análise fatorial confirmatória reportou que o modelo fatorial definido pelos autores, ajustado para uma amostra de 1908 professores, revelou uma qualidade de ajustamento razoável $(X 2=315.608 ; p=.000 ; X 2 / d f=24.301$; $C F I=$.952; $G F I=.956 ;$ RMSEA =. 1 15; P[rmsea<.05]<.000) (MAROCO, 2010). A avaliação da consistência interna da escala, considerando a amostra de 1908 professores, revelou, igualmente, valores indicadores de elevada consistência interna $(\alpha=.94)$, não sendo recomendada a eliminação de qualquer item dos 24 que constituem a escala.

\section{Procedimentos}

Após a validação dos instrumentos, procurou-se realizar todas as diligências formais para a obtenção de todas as autorizações para a recolha de dados em ambiente escolar nacional. $O$ pedido de autorização, feito à Direção-geral da Educação, para recolher dados junto dos diretores e professores das escolas públicas nacionais foi deferido com o Nº34600000 1.

Os instrumentos foram organizados numa plataforma online opensource, que permitiu a recolha e exportação de dados para aplicações de análise estatística e o convite de participação no estudo foi enviado aos dois grupos de participantes através do correio eletrónico institucional das escolas. No email enviado foi contextualizada toda a investigação a desenvolver e prestados todos os esclarecimentos com vista à garantia do consentimento informado por parte dos participantes. Foi ainda garantida a integridade dos 
dados e o anonimato na análise dos resultados. Terminado o processo de recolha de dados, procedeu-se, numa primeira fase, à exportação dos mesmos para uma aplicação de folha de cálculo para uma primeira organização e análise. Nesta primeira análise procurou-se eliminar submissões nulas, duplas submissões e outros dados irrelevantes para a investigação em causa. A base de dados resultante desta primeira análise foi importada para o software IBM SPSS Statistics (V.21) onde se desenvolveram todos os testes e análises estatísticas com vista à produção dos resultados.

No processo de recolha e análise de dados consideraram-se, ainda, os pressupostos definidos na Carta Ética para a Investigação em Educação e Formação do Instituto de Educação da Universidade de Lisboa, aprovada pela deliberação n. ${ }^{\circ} 453 / 2016$, de 15 de março, do conselho científico do Instituto de Educação da Universidade de Lisboa, e na Carta de Ética da Sociedade Portuguesa de Ciências da Educação (SPCE, 20 14).

\section{Participantes}

De modo a obter dados junto de um maior número de diretores de escolas e professores do ensino público nacional, decidiu-se inquirir toda a população constituída pelos diretores e professores dos 831 agrupamentos de escolas e escola não agrupadas existentes em Portugal Continental. A amostra constituída é formada pelos 133 diretores escolares e 1908 professores do ensino básico e secundário que responderam aos instrumentos de recolha de dados e aceitaram participar no estudo. Os diretores escolares são predominantemente do género masculino (52.63\%), têm entre 51 e 60 anos de idade, lecionam há mais de 20 anos (78.95\%) e apresentam mais de 11 anos de experiência na gestão e administração escolar (67.67\%). A maioria dos diretores frequentou formação especializada (pós-graduação e/ou mestrado) em gestão e administração escolar (75.10\%). Relativamente aos professores, a maioria é do género feminino (76.36\%), tem entre 41 e 50 anos de idade (42.19\%), leciona há mais de 21 anos (57.66\%) e trabalha na mesma escola há menos de 10 anos (53.51\%). Para análise dos dados cruzando as respostas de diretores e professores, procedeu-se à seleção de um subgrupo amostral considerando como participantes todos os diretores de escolas e agrupamentos de 
escolas com número de respostas de professores das suas escolas superiores a 10. $O$ grupo em causa foi constituído por 31 diretores 572 professores.

\section{Resultados}

O presente tópico organiza-se em torno dos principais resultados encontrados no estudo em causa. Assim, num primeiro foco de análise apresentaremos os resultados evidenciados pelos diretores escolares, num segundo tópico destacaremos os resultados evidenciados pelos professores do ensino básico e secundário e, por último, reportaremos a análise dos resultados cruzando os dois grupos de participantes.

\section{Diretores escolares}

O cálculo dos índices de autoeficácia ou proficiência e de utilização das tecnologias digitais pelos diretores escolares, nas suas práticas profissionais, operacionalizou-se através do somatório das opç̃oes de resposta selecionada pelos diretores em cada um dos itens das duas escalas aplicadas: a) Computer Self-efficacy Scale; e b) Escala de utilização das tecnologias na gestão escolar. Os resultados da tabela seguinte apresentam os valores médios dos scores médios totais e o desvio padrão para as duas escalas e para as cinco dimensões da escala de utilização das tecnologias na gestão escolar.

Os resultados evidenciam índices de autoeficácia $(M=3.95 ; S D=.48)$ e de utilização das tecnologias ( $M=4.22 ; S D=.64$ ) elevados, considerando que os valores em causa se poderiam situar entre o mínimo de 1 e máximo de 5 . Os scores apresentados em cada uma das dimensões da escala de utilização apresentam-se igualmente elevados. Para as variáveis em análise, considerou-se que valores compreendidos entre 1 e 2.4 pontos representam um nível baixo, entre 2.5 e 3.4 pontos representam um nível moderado ou favorável e valores entre 3.5 e 5 pontos representam um nível elevado. Constata-se ainda que é na dimensão relativa à gestão que os diretores apresentam um índice de utilização mais elevado ( $M=4.45 ; S D=.81)$. Em sentido oposto, o índice mais reduzido é apresentado na dimensão comunicação $(M=3.98 ; S D=.66)$, ainda que este se mantenha também elevado. 


\section{Tabela 1}

Valores médios e desvio padrão das escalas de proficiência e de utilização das tecnologias digitais

\begin{tabular}{|l|c|c|}
\hline \multicolumn{1}{|c|}{ Variáveis } & Média & Desvio padrão \\
\hline \hline Nível de Proficiência & 3.95 & .48 \\
\hline Utilização das tecnologias & 4.22 & .64 \\
\hline \hline \multicolumn{2}{|c|}{ Dimensões da escala de utilização das tecnologias digitais } \\
\hline Dimensão 1 - Comunicação & 3.98 & .66 \\
\hline Dimensão 2 - Planificação & 4.25 & .72 \\
\hline Dimensão 3 - Reuniões & 4.13 & .81 \\
\hline Dimensão 4 - Avaliação & 4.23 & .80 \\
\hline Dimensão 5 - Gestão & 4.45 & .81 \\
\hline
\end{tabular}

Com o objetivo de explorar a relação entre o nível de proficiência na utilização das tecnologias e os índices de utilização das mesmas na prática profissional dos diretores escolares, procedeu-se ao cálculo dos coeficientes de 15 correlação de Pearson (r) entre as variáveis, analisando tanto os scores totais das duas escalas como das cinco dimensões presentes na escala de utilização das tecnologias na gestão escolar.

A análise da tabela seguinte permite constatar a existência de correlação estatisticamente significativa entre todas as variáveis e que a mesma apresenta uma direção positiva, variando a sua intensidade entre baixa e elevada $(0.19<r<.90 ; p<0.01)$. De facto, encontramos uma correlação de intensidade moderada entre o score total de proficiência e o score total de utilização das tecnologias $(r=.33 ; p<.01)$, e entre o primeiro e todas as dimensões do segundo, sendo estas também estatisticamente significativas. 


\section{Tabela 2}

Coeficientes de correlação de Pearson entre o nível de proficiência, índice de utilização das tecnologias digitais e respetivas dimensões

\begin{tabular}{|c|c|c|c|c|c|c|}
\hline & $\begin{array}{l}\text { Utilização } \\
\text { das } \\
\text { tecnologias }\end{array}$ & $\begin{array}{c}\text { Dimensão } 1 \\
\text { Comunicaçãoo }\end{array}$ & $\begin{array}{l}\text { Dimensão } 2 \\
\text { Planificaçãao }\end{array}$ & $\begin{array}{c}\text { Dimensão } 3 \\
\text { Reuniões }\end{array}$ & $\begin{array}{c}\text { Dimensão } 4 \\
\text { Avaliação }\end{array}$ & $\begin{array}{c}\text { Dimensão } 5 \\
\text { Gestão }\end{array}$ \\
\hline Nível de Proficiência & $.33 * *$ & $.27^{* *}$ & $.39 * *$ & $.30 * *$ & $.31^{* \star}$ & $.19 *$ \\
\hline Utilização das tecnologias & & $.87^{* *}$ & $.77^{* \star}$ & $.82^{* *}$ & $.90 * *$ & $.85^{* *}$ \\
\hline Dimensão 1 Comunicação & & & $.61^{* *}$ & $.66^{* *}$ & $.72^{* *}$ & $.66^{* *}$ \\
\hline Dimensão 2 Planificação & & & & $.74^{* *}$ &, $59 * *$ & $.48^{* *}$ \\
\hline Dimensão 3 Reuniões & & & & & $.61^{* *}$ & .52 ** \\
\hline Dimensão 4 Avaliação & & & & & & $83^{* *}$ \\
\hline
\end{tabular}

\section{Professores do ensino básico e secundário}

A análise do índice de utilização das tecnologias digitais pelos professores, nas várias dimensões da sua prática profissional, obtido através das respostas dadas pelos 1908 professores aos 24 itens da Measure Teacher's Technology Use Scale revelou um valor médio de 4.31 e desvio padrão de .77, o que permite concluir que existe um elevado grau de utilização das tecnologias por parte dos professores, considerando a escala definida entre 1 e 5 . Consideram-se valores médios elevados aqueles que se apresentem superiores a 3.50, indicando um grau elevado de utilização das tecnologias nas referidas dimensões.

Através da tabela seguinte, verifica-se a existência de valores médios diferentes para cada uma das dimensões da escala. 
Análise da utilização das tecnologias digitais por diretores escolares e professores

\section{Tabela 3}

Valores médios e desvio padrão da escala de utilização das tecnologias digitais pelos professores

\begin{tabular}{|l|c|c|}
\hline & Média & Desvio padrão \\
\hline Índice de utilização das tecnologias (n=1908) & 4.31 & .77 \\
\hline Dimensão 1: Preparação de aulas & 4.53 & .64 \\
\hline Dimensão 2: E-mail profissional & 3.48 & .77 \\
\hline Dimensão 3: Instrução & 3.98 & .95 \\
\hline Dimensão 4: Adaptação às necessidades dos alunos & 3.83 & .92 \\
\hline Dimensão 5: Uso pelos alunos & 2.83 & 1.08 \\
\hline Dimensão 6: Suporte à produção dos alunos & 2.20 & 1.06 \\
\hline Dimensão 7: Avaliação & 4.17 & .91 \\
\hline
\end{tabular}

Assim, tendo em consideração as dimensões definidas na escala de utilização das tecnologias, acima mencionadas, é possível verificar diferenças nos valores médios apresentados em cada dimensão, encontrando-se o 17 valor médio mais reduzido $(M=2.20)$ na dimensão "suporte à produção dos alunos" e um valor médio mais elevado $(M=4.53)$ na dimensão "preparação de aulas". Por outro lado, contata-se que as dimensões que apresentam valores médios mais elevados ao nível da utilização das tecnologias foram, por ordem decrescente, ao nível da preparação de aulas $(M=4.53)$, avaliação dos alunos $(M=4.20)$, instrução $(M=3.98)$ e utilização do email profissional $(M=3.50)$.

Em sentido oposto, verifica-se que as dimensões 'uso pelos alunos' e 'suporte à produção dos alunos' apresentam valores médios de 2.82 e 2.20, respetivamente. Os baixos valores médios apresentados são indicadores de baixos índices de utilização das tecnologias por parte dos professores nas atividades associadas a estas dimensões. Se considerarmos a diferença entre a dimensão que apresentou o maior valor médio e a que apresentou o menor valor médio, respetivamente 'preparação de aulas' e 'suporte à produção dos alunos', é possível verificar que se regista uma diferença assinalável de 2.33 pontos numa escala de apenas 5 pontos. 


\section{Diretores escolares e professores}

Um dos objetivos do estudo consistiu na exploração de possível associação entre os scores médios apresentados pelos diretores e os scores de utilização das tecnologias apresentados pelos professores das suas escolas. Como referimos anteriormente, para o cruzamento dos resultados consideraram-se todos os diretores de escolas e agrupamentos de escolas com número de professores respondentes das suas escolas superiores a 10, no total 31 diretores e 572 professores.

A tabela seguinte apresenta os scores médios de utilização das tecnologias evidenciados pelo grupo de professores de cada uma das 31 escolas e os scores de utilização das tecnologias e scores de proficiência do respetivo diretor.

\section{Tabela 4}

Valores médios das escalas de utilização das tecnologias digitais pelos professores e utilização das tecnologias digitais e proficiência dos diretores escolares

\begin{tabular}{|l|c|c|c|c|}
\hline Escola & n Professores & $\begin{array}{c}\text { Score médio de utilização } \\
\text { das tecnologias dos profes- } \\
\text { sores da escola }\end{array}$ & $\begin{array}{c}\text { Score de uti- } \\
\text { lização das } \\
\text { tecnologias do } \\
\text { Diretor }\end{array}$ & $\begin{array}{c}\text { Score de profici- } \\
\text { ência do Diretor }\end{array}$ \\
\hline \hline Escola 1 & 10 & 3.31 & 3.76 & 4.44 \\
\hline Escola 2 & 19 & 3.17 & 3.52 & 3.59 \\
\hline Escola 3 & 12 & 3.67 & 4.52 & 4.15 \\
\hline Escola 4 & 10 & 3.46 & 4.14 & 3.63 \\
\hline Escola 5 & 27 & 3.58 & 4.67 & 3.52 \\
\hline Escola 6 & 14 & 3.46 & 2.05 & 4.11 \\
\hline Escola 7 & 21 & 4.13 & 4.48 & 4.04 \\
\hline Escola 8 & 12 & 3.54 & 4.52 & 3.67 \\
\hline Escola 9 & 22 & 3.67 & 3.76 & 3.74 \\
\hline Escola 10 & 23 & 3.42 & 4.71 & 4.67 \\
\hline Escola 11 & 13 & 3.71 & 4.33 & 3.93 \\
\hline Escola 12 & 15 & 3.50 & 4.10 & 3.74 \\
\hline Escola 13 & 17 & 3.38 & 5.00 & 4.26 \\
\hline Escola 14 & 15 & 3.67 & 4.76 & 2.56 \\
\hline Escola 15 & 14 & 3.69 & 4.19 & 4.26 \\
\hline \hline Escola 16 & 13 & 3.58 & 3.26 \\
\hline \hline
\end{tabular}


Análise da utilização das tecnologias digitais por diretores escolares e professores

\section{Tabela 4}

Valores médios das escalas de utilização das tecnologias digitais pelos professores e utilização das tecnologias digitais e proficiência dos diretores escolares (Continuação)

\begin{tabular}{|c|c|c|c|c|}
\hline Escola & n Professores & $\begin{array}{c}\text { Score médio de utilização } \\
\text { das tecnologias dos profes- } \\
\text { sores da escola }\end{array}$ & $\begin{array}{c}\text { Score de uti- } \\
\text { lização das } \\
\text { tecnologias do } \\
\text { Diretor }\end{array}$ & $\begin{array}{c}\text { Score de profici- } \\
\text { ência do Diretor }\end{array}$ \\
\hline \hline Escola 17 & 22 & 3.04 & 4.33 & 4.63 \\
\hline Escola 18 & 16 & 3.31 & 4.10 & 3.89 \\
\hline Escola 19 & 18 & 3.44 & 4.86 & 3.85 \\
\hline Escola 20 & 20 & 3.21 & 4.71 & 4.85 \\
\hline Escola 21 & 29 & 3.54 & 3.86 & 3.63 \\
\hline Escola 22 & 39 & 3.42 & 4.10 & 4.44 \\
\hline Escola 23 & 24 & 3.98 & 4.71 & 3.67 \\
\hline Escola 24 & 14 & 3.61 & 4.57 & 3.89 \\
\hline Escola 25 & 23 & 3.38 & 4.33 & 3.89 \\
\hline Escola 26 & 41 & 3.46 & 2.33 & 2.33 \\
\hline Escola 27 & 12 & 3.34 & 4.62 & 4.63 \\
\hline Escola 28 & 14 & 3.86 & 3.76 & 3.74 \\
\hline Escola 29 & 11 & 3.75 & 4.67 & 3.44 \\
\hline Escola 30 & 12 & 3.88 & 4.33 & 4.15 \\
\hline Escola 31 & 20 & 3.52 & 3.62 & 3.96 \\
\hline
\end{tabular}

De seguida, procurou-se analisar a existência de relação entre os scores registados de índice de utilização das tecnologias e nível de proficiência dos diretores e os correspondentes índices de utilização das tecnologias evidenciados pelos professores a lecionar nas suas escolas, pelo que os valores registados para cada escola se encontram representados na tabela 5.

De igual modo, procedeu-se ao cálculo do coeficiente de correlação de Pearson entre o score de utilização das tecnologias pelos professores e o score de proficiência e score de utilização das tecnologias dos respetivos diretores das suas escolas, como representado na tabela seguinte. Analisando os dados da tabela seguinte, constata-se a existência de fracos coeficientes de correlação (sem significância estatística) entre as variáveis. Como exceção 
encontra-se o índice de utilização das tecnologias apresentado pelos professores da escola 20 e nível de proficiência do respetivo diretor, que apresenta um coeficiente de correlação assinalável ( $r=.51)$.

Tabela 5

Coeficientes de correlação de Pearson entre o nível de utilização das tecnologias digitais pelos professores e o índice de utilização das tecnologias digitais e nível de proficiência do respetivo diretor

\begin{tabular}{|c|c|c|c|c|c|c|}
\hline \multicolumn{3}{|c|}{ Score de proficiência do Diretor } & \multicolumn{4}{|c|}{ Score de utilização das tecnologias do Diretor } \\
\hline & Escola & n Professores & $\begin{array}{l}\text { Pearson } \\
\text { Coeficiente }\end{array}$ & Sig. & Pearson Coeficiente & Sig. \\
\hline \multirow{23}{*}{ 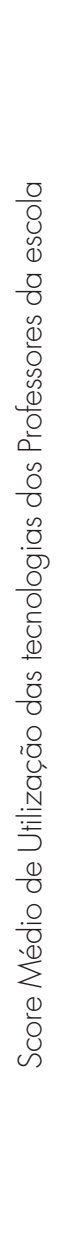 } & Escola 1 & 10 & .19 & .41 & .46 & 3.89 \\
\hline & Escola 2 & 19 & .40 & .09 & .16 & 3.85 \\
\hline & Escola 3 & 12 & .35 & 27 & .18 & 4.85 \\
\hline & Escola 4 & 10 & .00 & .99 & .17 & 3.63 \\
\hline & Escola 5 & 27 & .35 & .08 & .18 & 4.44 \\
\hline & Escola 6 & 14 & .41 & .14 & .27 & 3.67 \\
\hline & Escola 7 & 21 & .34 & .16 & .32 & 3.89 \\
\hline & Escola 8 & 12 & .37 & .25 & .37 & 3.89 \\
\hline & Escola 9 & 22 & .23 & .31 & .13 & 2.33 \\
\hline & Escola 10 & 23 & .18 & .36 & .16 & 4.63 \\
\hline & Escola 11 & 13 & .14 & .64 & .01 & 3.74 \\
\hline & Escola 12 & 15 & .19 & .47 & .08 & 3.44 \\
\hline & Escola 13 & 17 & .17 & .70 & .21 & 4.15 \\
\hline & Escola 14 & 15 & .38 & .16 & .04 & 3.96 \\
\hline & Escola 15 & 14 & .13 & .66 & .07 & \\
\hline & Escola 16 & 13 & .10 & .75 & .01 & \\
\hline & Escola 17 & 22 & .11 & .62 & .06 & \\
\hline & Escola 18 & 16 & .18 & .65 & .09 & \\
\hline & Escola 19 & 18 & .19 & .56 & .10 & \\
\hline & Escola 20 & 20 & $.51 *$ & .02 & .11 & \\
\hline & Escola 21 & 29 & .19 & .34 & .18 & \\
\hline & Escola 22 & 39 & .24 & .30 & -.07 & \\
\hline & Escola 23 & 24 & .10 & .65 & .05 & \\
\hline
\end{tabular}


Análise da utilização das tecnologias digitais por diretores escolares e professores

Tabela 5

Coeficientes de correlação de Pearson entre o nível de utilização das tecnologias digitais pelos professores e o índice de utilização das tecnologias digitais e nível de proficiência do respetivo diretor (Continuação)

\begin{tabular}{|c|c|c|c|c|c|c|}
\hline \multicolumn{3}{|c|}{ Score de proficiência do Diretor } & \multicolumn{4}{|c|}{ Score de utilização das tecnologias do Diretor } \\
\hline & Escola & n Professores & $\begin{array}{c}\text { Pearson } \\
\text { Coeficiente }\end{array}$ & Sig. & Pearson Coeficiente & Sig. \\
\hline \multirow{8}{*}{ 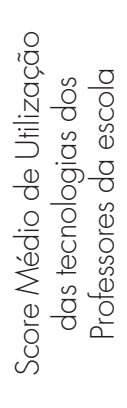 } & Escola 24 & 14 & .51 & .09 & .46 & .09 \\
\hline & Escola 25 & 23 & 25 & .31 & 23 & .29 \\
\hline & Escola 26 & 41 & .24 & .14 & 24 & .14 \\
\hline & Escola 27 & 12 & .49 & .09 & 39 & .21 \\
\hline & Escola 28 & 14 & .30 & .30 & 25 & .39 \\
\hline & Escola 29 & 11 & .34 & .30 & .06 & .86 \\
\hline & Escola 30 & 12 & .38 & .22 & .17 & .60 \\
\hline & Escola 31 & 20 & .39 & .19 & .15 & .54 \\
\hline
\end{tabular}

* Significativo para $\alpha=0.05$

Com o objetivo de identificar padrões de semelhança entre as várias escolas procedeu-se ainda à análise exploratória de clusters. $\bigcirc$ processo de identificação de clusters sustentou-se no método de agrupamento hierárquico de clusters com base em variáveis (Nearest Neighbor). Previamente e com vista a sustentar o processo de criação dos clusters realizou-se o teste estatístico ANOVA entre o score médio de utilização das tecnologias pelos professores de cada escola e o score médio de autoeficácia do diretor ( $F=2.24$, sig=.002), e entre o score médio de utilização das tecnologias pelos professores de cada escola e o score médio de utilização das tecnologias do diretor $(F=1.52$, sig=.38). Considerando os resultados da ANOVA estabeleceu-se a identificação de clusters com base no score médio de proficiência apresentado pelo diretor, na medida em que só esta variável relevou significância estatística. Foi possível organizar três clusters com semelhanças estatisticamente significativas, a saber: (i) escolas cujos diretores apresentam baixos índices de proficiência, (ii) escolas cujos diretores apresentam índices moderados de proficiência e (iii) escolas cujos diretores apresentam elevados índices de proficiência. A tabela 6 apresenta a organização dos clusters encontrados, sendo que o gráfico da figura seguinte sistematiza, de forma visual, mais discriminadamente essa informação. 


\section{Tabela 6}

Análise de Clusters com base no score médio de proficiência do Diretor e o score médio de utilização das tecnologias dos respetivos professores da escola

\begin{tabular}{|c|c|c|c|c|}
\hline \multirow{2}{*}{ Escola } & \multirow{2}{*}{$\mathrm{n}$} & \multicolumn{3}{|c|}{ Subset for alpha $=0.05$} \\
\hline & & 1 & 2 & 3 \\
\hline Escola 26 & 41 & -1.18 & & - \\
\hline Escola 14 & 15 & -1.170 & & - \\
\hline Escola 16 & 13 & -.45 & & - \\
\hline Escola 29 & 11 & - & -.23 & - \\
\hline Escola 23 & 24 & - & -.07 & - \\
\hline Escola 5 & 27 & - & -.06 & - \\
\hline Escola 8 & 12 & - & -.05 & - \\
\hline Escola 7 & 21 & - & -.01 & - \\
\hline Escola 11 & 13 & - & .02 & - \\
\hline Escola 21 & 29 & - & .13 & - \\
\hline Escola 24 & 14 & - & .23 & - \\
\hline Escola 9 & 22 & - & .23 & - \\
\hline Escola 12 & 15 & - & .25 & - \\
\hline Escola 31 & 20 & - & .29 & - \\
\hline Escola 4 & 10 & - & .30 & - \\
\hline Escola 2 & 19 & - & .33 & - \\
\hline Escola 30 & 12 & - & & - \\
\hline Escola 19 & 18 & - & & - \\
\hline Escola 18 & 16 & & .44 & \\
\hline Escola 25 & 23 & - & .45 & \\
\hline Escola 3 & 12 & & & \\
\hline Escola 27 & 12 & & & \\
\hline Escola 15 & 14 & - & - & \\
\hline Escola 28 & 14 & & & \\
\hline
\end{tabular}


Tabela 6

Análise de Clusters com base no score médio de proficiência do Diretor e o score médio de utilização das tecnologias dos respetivos professores da escola (Continuação)

\begin{tabular}{|c|c|c|c|c|}
\hline \multirow{2}{*}{ Escola } & \multirow{2}{*}{$\mathrm{n}$} & \multicolumn{3}{|c|}{ Subset for alpha $=0.05$} \\
\hline & & 1 & 2 & 3 \\
\hline Escola 6 & 14 & & - & - \\
\hline Escola 13 & 17 & - & - & \\
\hline Escola 22 & 39 & - & - & \\
\hline Escola 1 & 10 & - & - & 1.18 \\
\hline Escola 10 & 23 & - & - & 1.29 \\
\hline Escola 17 & 22 & - & - & 1.54 \\
\hline Escola 20 & 20 & - & - & 1.61 \\
\hline $\begin{array}{c}\text { Significância } \\
\text { Estatística }\end{array}$ & & .19 & .34 & .87 \\
\hline
\end{tabular}

Figura 1

Box plot dos clusters identificados com base na matriz de proximidade

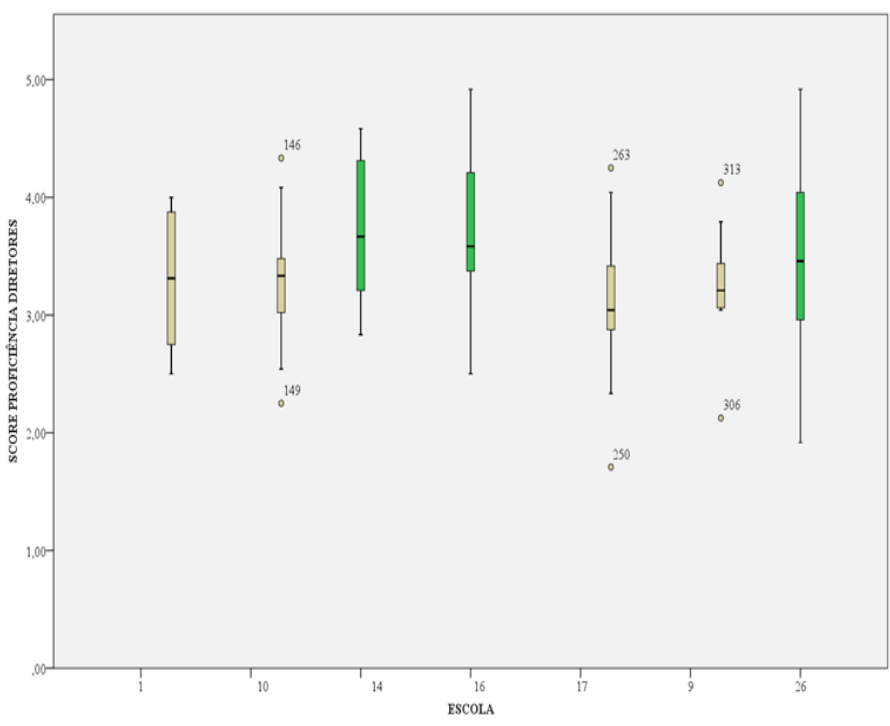

Diretores com Elevada Probckincia 
Pela análise dos resultados, infere-se que nos clusters de escolas organizados, verifica-se existência de distribuições semelhantes no que se refere ao índice de autoeficácia do diretor e ao nível de utilização das tecnologias por parte dos professores que integram o corpo docente da sua instituição.

\section{Conclusões}

presente estudo foi desenvolvido junto de 133 diretores escolares e 1908 professores do ensino básico e secundário. Numa primeira fase, analisaram-se os níveis de proficiência e de utilização das tecnologias digitais por parte de diretores de escolas e agrupamentos de escolas, do ensino público português, nas práticas de gestão escolar. De seguida, analisou-se o índice de utilização das tecnologias digitais apresentado pelos professores em várias dimensões da sua prática e atividade profissional. Por fim, procurou-se a existência de algum tipo de relação entre os níveis de proficiência e utilização das tecnologias apresentado pelos diretores escolares e o índice de utilização das mesmas apresentado pelos professores das suas escolas.

Os resultados permitiram concluir que os diretores escolares portugueses apresentam um grau de proficiência e um índice de utilização das tecnologias favoráveis nas várias dimensões da sua atividade profissional. Constatou-se, contudo, que a utilização de formas de comunicação com recurso às novas tecnologias, (como forma privilegiada de contacto entre os vários agentes educativos pais, professores, alunos e órgão de gestão), apresenta valores mais reduzidos pelo que se entende esta como uma dimensão que valerá a pena estimular e desenvolver dada a usa importância e pertinência atuais.

A análise da relação entre o grau de proficiência e o índice de utilização das tecnologias pelos diretores escolares revelou que as variáveis se correlacionam de forma expressiva e estatisticamente significativa. Assim, pode inferir-se que a autoeficácia no uso das tecnologias ou grau de proficiência se apresenta associada à utilização das tecnologias nas múltiplas dimensões da atividade profissional dos diretores escolares. Desta forma, constata-se que para se incentivarem melhores e mais regulares práticas de utilização das tecnologias na gestão escolar será, igualmente, necessário atender às atitudes 
Análise da utilização das tecnologias digitais por diretores escolares e professores

pessoais dos diretores escolares, nomeadamente aquelas que se associam ao seu sentido de autoeficácia e competência no uso das mesmas.

Os resultados extraídos do estudo encontram-se alinhados com vários estudos internacionais que salientam, igualmente, a importância das atitudes, competência e utilização das tecnologias pelos diretores escolares, enquanto fatores de influência na adoção e implementação das tecnologias digitais nas suas escolas (ABDULLAH, DEWITT, ALIAS, 2013; GRAY, SMITH, 2007; MAKI, 2008; ZAINALLY, 2008).

Num segundo momento, procedeu-se à análise da utilização das tecnologias digitais pelos professores em várias dimensões da sua prática profissional. A análise das respostas dos 1908 professores revelou um índice elevado de utilização, o que evidencia uma forte utilização das tecnologias na sua atividade enquanto docentes. Olhando de forma independente para os índices de utilização das tecnologias em cada uma das sete dimensões analisadas da escala utilizada, verificou-se que os valores mais elevados se referiam especificamente a atividades associadas à 'Preparação de Aulas' e à 'Avaliação'. Estes resultados revelam que os professores de tendem a apresentar índices de utilização das tecnologias elevados em (i) tarefas de planificação 25 de aulas, elaboração materiais didáticos e de recursos educativos para suporte às atividades de sala de aula, construção de fichas e atividades a disponibilizar aos alunos; e (ii) atividades relacionadas com a avaliação, como sejam a elaboração e correção de instrumentos de avaliação (testes, enunciados de propostas de trabalho), cálculo e registo das classificações, preenchimento de grelhas de observação e de outros recursos para avaliação.

Em sentido oposto, observaram-se índices de utilização mais reduzidos nas dimensões associados com 'Uso das Tecnologias pelos Alunos em Sala de Aula' e ao 'Suporte à Produção dos Alunos'. Os dados evidenciam que a utilização das tecnologias pelos alunos, em sala de aula, para a realização de atividades de aprendizagem e produção de conhecimento não se encontra ainda nos níveis ambicionados, indiciando que a efetiva integração curricular das tecnologias está ainda por conseguir.

Os resultados encontrados para as várias dimensões da escala encontram concordância com outros estudos nacionais que, recorrentemente, têm vindo a evidenciar que os professores tendem a usar mais as tecnologias como suporte ao desenvolvimento do seu trabalho de preparação de aulas, 
construção de recursos didáticos e avaliação dos alunos em detrimento da sua integração no trabalho direto com/ e dos alunos em sala de aula (ALVES, ABRANTES, RODRIGUES, DIAS, 2013 ; PEDRO, 2011 ; SANTOS, PEDRO, 2015; PIEDADE, 2010; PIEDADE, PEDRO, 2011 ; 20121.

Por último, procedeu-se à análise conjunta das variáveis analisadas junto de diretores escolares e professores. A análise do cruzamento entre os scores registados junto dos diretores escolares e dos professores das suas respetivas escolas (num total de 31 diretores e 572 professores) permitiu verificar que, no global, não é possível identificar uma associação estatisticamente significativa entre estas variáveis. No entanto, uma análise subsequente permitiu organizar as escolas com base no nível de proficiência tecnológica evidenciada pelos seus diretores e detetar que no seio destas, o comportamento de utilização das tecnologias evidenciado pelos seus professores se revela semelhante ao nível de proficiência revelado pelo diretor respetivo. Assim, encontramos evidência de um índice de proficiência do diretor reduzido quando se verifica um índice de utilização das tecnologias pelos professores também reduzido e, em oposição, revela-se o primeiro elevado quando o segundo também se revela elevado. Confirma-se, deste modo, a importância do papel do diretor escolar alicerçado nas diferenças encontradas nos grupos de escolas analisados.

Concluindo, o processo de adoção e integração efetiva das tecnologias digitais em contexto escolar requer a conjugação favorável de vários fatores envolvendo professores, diretores escolares, alunos, encarregados de educação e decisores políticos. Importa, igualmente, referir que este processo apenas pode ter sucesso se ao descrito anteriormente juntarmos a existência de professores inovadores e competentes na utilização das tecnologias em contexto educativo, currículos flexíveis, diretores inovadores e visões e culturas de escola que privilegiem a utilização das tecnologias e a inovação nos seus contextos.

\section{Referências}

ALVES, Nuno de Almeida; ABRANTES, Pedro; RODRIGUES, Carla; DIAS, Paulo Coelho. TIC no ensino secundário: usos e mediações. Forum Sociológico, Lisboa, v. 23, p. 87-95, 2013. 
ABDULLAH, Nur Ain Wong; DEWITT, Dorothy; ALIAS, Norlidah. School improvement efforts and challenges: A case study of a principal utilizing information communication technology. Procedia - Social and Behavioral Sciences, Sakarya, v. 103, p. 791-800, nov. 2013.

AFSHARI, Moigan; GHAVIFEKR, Simin; SIRAJ, Saedah; SAMAD, Sukor Ab. Transformational leadership role of principals in implementing informational and communication technologies in schools. Life Science Journal, Zhengzhou, v. 9, n. 1, p. 281-284, jan. 2012.

AFSHARI, Moigan; BAKAR, Kamariah Abu; LUAN, Wong Su; FOOI, Foo Say. Computer use by school principals. Turkish Online Journal of Educational Technology, Sakarya, v. 9, n. 3, p. 8-25, jul. 2010.

ALMEIDA, Leandro; FREIRE, Teresa. Metodologia de investigação em psicologia e educação. Braga: Psiquilibrios Edições, 2008.

ANDERSON, Ronald; DEXTER, Sara. School technology leadership: An empirical investigation of prevalence and effect. Educational Administration Quarterly, Utah, v. 41 n. 1, p. 49-82, fev. 2005 .

BEBELL, Damian; RUSSELL, Michael; O'DWYER, Laura. Measuring teachers' technology uses: Why multiple-measures are more revealing. Journal of Research on Technology in Education, Florida, v. 37, n. 1, p. 45-63, fev. 2004.

CAKIR, Recep. Technology integration and technology leadership in schools as learning organizations. The Turkish Online Journal of Educational Technology, Sakarya, v, 1 1, n. 4, p. 273-282, out. 2012.

CAKIR, Turan. The attitudes of preschool teachers and principals towards computer using. Anthropologist, Haryana, v. 18, n. 3, p. 735-744, out. 2014.

CALABRESE, Raymond. The school leader's imperative: leading change, International Journal of Educational Management, Bingley, v. 16, n. 7, p. 326-332, 2002.

CASSIDY, Simon; EACHUS, Peter. Developing the computer user self-efficacy (CUCE) scale: Investigating the relationship between computer self-efficacy, gender and experience with computers. Journal of Educational Computing Research, California, v. 26, n. 2, p. 133154, mar. 2002.

CRESWELL, John. Projeto de pesquisa. Métodos qualitativo, quantitativo e misto. 3. ed. Porto Alegre: Artmed, 2010.

DAY, Christopher; LEITHWOOD, Kenneth. Successful principal leadership in times of change: An international perspective. Dordrecht, UK: Springer, 2007. 
DRENT, Marjolein; MEELISSEN, Martina. Which factors obstruct or stimulate teacher educators to use ICT innovatively?. Computers \& Education, Amsterdam, v. 51, p. 187-199, jun. 2008.

GASTELÚ, Carlos Arturo Torres; KISS, Gabor; DOMINGUEZ, Agustin Lagunes. ITC integration process in basic education: the case of Veracruz city in Mexico. Procecia - Social and Behavioral Sciences, Sakarya, v. 176, p. 819-823, mar. 2015.

GRAY, David; SMITH, Agnes. Case studies in 21 st century school administration: addressing challenges for educational leadership. USA: Sage Publications, Inc, 2007.

HOQUE, Kazi Enamul; RAZAK, Ahmad Zabidi Abdul; ZOHORA, Mosa Fatema. ICT Utlilization among school teachers and principals in Malaysia. International Journal of Academic Research in Progressive Education and Development, Bahawalpur, v. 1, n. 4, p. 17-34, 2012.

JACOBSON, Michael; HUNTER, William. Leadership and technology in schools. 2004. Disponível em: http://www.ucalgary.ca/ iejll/volume7/leadershipandtechnologyeditorial. html. Acesso em: 16 jun. 2017.

KHALID, Saifuddin; BUUS, Lilian. A theoretical framework mapping barriers of integration and adopting educational technology. Research and Practice in Technology Enhanced Learning, Singapore, v. 1, n. 1, p. 1-19, jul. 2014.

MAKEWA, Lazarus; MEREMO, Jackson; ROLE, Elizabeth; ROLE, Jesse. ICT in secondary school administration in rural Southern Kenya: an Educator's eye on its importance and use. International Journal of Education and Development Using Information and Communication Technology, Barbados, v. 9, n. 2, p. 48-63, 2013.

MAKI, Christiana. Information and communication technology for administration and management for secondary schools in Cyprus. Journal of Online Learning and Teaching, California, v. 4, n. 3, p. 18-20, 2008.

MAROCO, João. Análise estatística com utilização do SPSS. 3. ed. Lisboa: Edições Sílabo, 2010.

MAROCO, João. Análise estatística com utilização do SPSS. 4. ed. Lisboa: Edições Sílabo, 2011 .

MOOLENAAR, Nienke; SLEEGERS, Peter. The networked principal: Examining principals' social relationships and transformational leadership in school and district networks. Journal of Educational Administration, Bingley, v. 53, n. 1, p. 8-39, 2015. 
PEDRO, Neuza. Utilização educativa das tecnologias, acesso, formação e auto-eficácia dos professores. 2011 . 392f. Tese (Doutoramento em Educação) - Programa de Doutoramento em Educação na especialidade de Tecnologias da Informação e Comunicação na Educação, Instituto de Educação da Universidade de Lisboa, Lisboa, 2011.

PEDRO, Neuza; PIEDADE, João. Efeitos da formação na autoeficácia e na utilização educativas das TIC pelos professores: estudo das diferenças entre os regimes formais e informais de formação. Revista e-curriculum, São Paulo, v. 11, n. 2, p. 766-793, set./dez. 2013.

PELED, Yehuda; KALID, Yael; DORI, Yehudit Judy. School principals' influence on science teachers' technology implementation: a retrospective analysis. Journal of Leadership in Education, Washington, v. 14, n. 2, p. 229-245, 2011.

PIEDADE, João. Utilização das tecnologias pelos professores de uma escola do ensino básico e secundário. 2010. 243f. Dissertação (Mestrado em Tecnologias e Metodologias em E-learning) - Faculdade de Ciências da Universidade de Lisboa, Lisboa, 2010.

PIEDADE, João; PEDRO, Neuza. Efeitos da formação no sentido de autoeficácia e na utilização educativa das TIC: estudo com um grupo de professores do ensino básico e secundário. In: CONFERÊNCIA INTERNACIONAL CHALLENGES; 2011 . CONFERÊNCIA 292011.

RUSSELL Michael; BEBELL Damian; O'DWYER Laura. Measuring teachers' technology uses: Why multiple-measures are more revealing. Journal of Teacher Education, Michigan, v. 37 , n. 1, p.45-63. 2004.

SAITI, Anna; PROKOPIADOU, Georgia. Impact of information and communication technologies on school administration: Research on the Greek schools of secondary education. Lecture Notes in Computer Science, Heidelberg, v. 5693, p. 305-316, 2009.

SANTOS, Ana Paula. Um estudo longitudinal sobre o efeito da formação no índice de autoeficácia e nos níveis de utilização das TIC dos professores. 2015. 217f. Tese (Doutoramento em Educação) - Programa de Doutoramento em Educação na especialidade de Tecnologias da Informação e Comunicação na Educação, Instituto de Educação da Universidade de Lisboa, Lisboa, 2015.

SEYAL, Afzaal. Examining the role of transformational leadership in technology adoption: Evidence from Bruneian technical \& vocational establishments (TVE). Journal of Education and Practice, Hong Kong, v. 6, n. 8, p. 32-43, 2015. 
SCHILLER, John. Working with ICT: Perceptions of Australian principals. Journal of Educational Administration, Bingley, v. 41, n. 2, p. 171-185, 2003.

STUART, Lindsay; MILLS, Annet; REMUS, Ulrich. School leaders, ICT competence and championing innovations. Computers \& Education, Amsterdam, v. 52, p. 733-74 1, nov. 2009.

TONDEUR, Jo; KEER, Hilde van; BRAAK, Johan van; VALCKE, Martin. ICT integration in the classroom: Challenging the potential of a school policy. Computers \& Education, Amsterdam, v. 51, n. 1, p. $212-223$, ago. 2008.

WONG, Emily; LI, Sandi. Framing ICT implementation in a context of educational change: A multilevel analysis. School Effectiveness and School Improvement, London, v. 19, n. 1, p. 99-120, maio. 2008.

ZAINALLY, Hossein. Administration of faculties by information and communication technology and its obstacles. International Journal of Education and Information Technologies, Victoria - British Columbia, v. 2, n. 1, p. 24-30, 2008

Prof. Dr. João Piedade Instituto de Educação (UIDEF) Universidade de Lisboa (Portugal) Grupo de Investigação "Educação Tecnologia e Sociedade" E-mail: jmpiedade@ie.ulisboa.pt

Profa. Dra. Neuza Pedro Instituto de Educação (UIDEF) Universidade de Lisboa (Portugal) Grupo de Investigação "Educação Tecnologia e Sociedade" E-mail:nspedro@ie.ulisboa.pt Recebido 7 nov. 2018 Aceito 4 jan. 2019 